\title{
Abordaje de problemas crónicos en atención primaria mediante el modelo tareas orientadas a los procesos de cuidado (TOPIC)
}

Approaching chronic problems in primary-care using the task-oriented processes in care (TOPIC)

\author{
Jojay S Ramal-Moreno ${ }^{1, a}$, Dayana Urday-Fernández ${ }^{2, a}$, Miguel Ricapa-Guerrero ${ }^{3, b}$, \\ Renato Sánchez-Salazar ${ }^{3, b}$, María S Cuba-Fuentes ${ }^{3, c}$
}

\section{RESUMEN}

El cuidado integral de las personas demanda un entrenamiento para atender problemas crónicos frecuentes en la consulta ambulatoria. Los casos de hipertensión, diabetes mellitus y asma pueden ser abordados mediante modelos de atención en la práctica ambulatoria del primer nivel de atención; uno de ellos es el modelo TOPIC (del inglés: Task-Oriented Processes in Care), el cual permite ordenar la consulta en una secuencia estructurada de cuatro tareas mayores: procesamiento de la información, desarrollo de una adecuada relación médico-paciente-familia, integración de la información y aprendizaje de por vida. El procesamiento de la información contempla cuatro sub-actividades en las que se evalúa las expectativas y preocupaciones del paciente, la gravedad y el control de las condiciones, la adherencia y los efectos adversos del tratamiento, se analiza los daños de órgano blanco propios de la enfermedad y se revisan las comorbilidades. La aplicación de este modelo puede ayudar a los médicos y sus pacientes a ser más eficientes en el manejo de problemas crónicos, teniendo en cuenta el poco tiempo del que se dispone en una consulta ambulatoria.

PALABRAS CLAVE: Enfermedad crónica, atención primaria de salud, atención ambulatoria. (Fuente: DeCS BIREME).

\section{SUMMARY}

The integrative approach to care for patients demands training to evaluate frequent chronic problems in the outpatient clinic. Patients with hypertension, diabetes mellitus and asthma can be approach using models of evaluations such as the TOPIC that allows to order the evaluations in a structured sequence of four mayor tasks: processing of the information, development of an adequate physician-patient-family relationship and integrating information and learning for life. Processing information implies four sub-activities in which we evaluate patient's expectations and concerns, severity and control of conditions, adherence and side effects of treatments focusing on damage to target organs and comorbidities. Applying this model can help physicians and their patients to be more efficient in managing chronic problems considering the short time available for ambulatory care.

KEYWORDS: Chronic disease, primary health care, ambulatory care. (Source: MeSH NLM).

\footnotetext{
Policlínico Guillermo Kaelin de la Fuente. Lima, Perú.

Centro de salud mental comunitario. Lima, Perú.

Universidad Peruana Cayetano Heredia. Lima, Perú.

Médico de familia;

Médico Residente de Medicina Familiar y Comunitaria;

Profesora auxiliar
} 


\section{INTRODUCCIÓN}

En las últimas décadas se ha visto un cambio significativo en el patrón de las principales causas de mortalidad, caracterizado por un incremento significativo de las muertes por enfermedades crónicas (1). En 1990 el 57\% del total de muertes se debieron a enfermedades crónicas no transmisibles (2), y en el año 2017 éstas representaron el 73,4\% de las muertes y el $53 \%$ del total de años de vida perdidos (3). Las enfermedades crónicas como la diabetes mellitus, enfermedades cardiovasculares y respiratorias impondrán una carga aún mayor en el futuro (4).

En países con ingresos medios como Perú, se ha producido la denominada transición demográfica (5), con disminución de las tasas totales de fecundidad, disminución de la mortalidad infantil y aumento de la esperanza de vida al nacer (2). Estos cambios repercuten en la composición de la estructura de la población, en su envejecimiento y morbilidad (6).

Así mismo, la creciente tendencia al envejecimiento de la población tiene enormes implicaciones económicas para los países $(1,7)$, desde el incremento requerido de gastos en infraestructura y tecnología sanitaria, como el costo generado por pérdida de productividad por discapacidad o muerte prematura (8).

La mortalidad prematura es significativa en cuatro categorías principales de enfermedades crónicas no transmisibles: enfermedad cardiovascular, cáncer, enfermedad pulmonar obstructiva crónica y diabetes mellitus, representando en conjunto la mayoría de las muertes en personas de 45 años de edad a más (9).

Una de cada tres muertes en el mundo se deben a enfermedades cardiovasculares, lo que significa $43 \%$ de las muertes por enfermedades crónicas no transmisibles (3), a pesar de la disponibilidad de tratamientos eficaces, económicos y seguros; siendo los países de ingresos medios y bajos los que experimentan un incremento importante de estas muertes prematuras (9).

Cerca de 65 millones de personas sufren de enfermedad pulmonar obstructiva crónica (EPOC) y tres millones mueren cada año, siendo la tercera causa de muerte en todo el mundo. El asma es la enfermedad crónica más común de la niñez, afectando al 14\% de esta población, con cerca de 334 millones de personas con esta condición (10).
Se estima que hay más de 500 millones de casos prevalentes de diabetes tipo 2 en todo el mundo. La prevalencia mundial de la diabetes se ha duplicado en los últimos 25 años, de $4,7 \%$ a $8,8 \%$, siendo los países de ingresos medios bajos los que han experimentado un incremento más acelerado además de un mayor porcentaje de personas no diagnosticadas (11).

Por ello, la Organización Mundial de la Salud (OMS) y otros organismos han resaltado la importancia de fortalecer la respuesta del sistema de salud a las enfermedades no transmisibles en general, con énfasis en la diabetes mellitus, particularmente a nivel de atención primaria, aplicando directrices y protocolos para mejorar el diagnóstico y la gestión de la diabetes en la atención primaria de salud (12).

\section{Atención primaria de salud y sistemas sanitarios frente a enfermedades crónicas}

En el abordaje de enfermedades crónicas no trasmisibles, los sistemas sanitarios se organizan en tres ejes prioritarios: acciones de promoción de salud, acciones de vigilancia evaluación y monitoreo, y provisión de cuidado integral a las personas con enfermedades crónicas (13) (tabla 1).

El primer nivel de atención debería ser aquel al que los ciudadanos tienen acceso fácil evitando barreras ante sus necesidades de salud, en el que pueden ser atendidos por un equipo multidisciplinario; en este nivel se deben brindar cuidados integrales respondiendo a las necesidades de promoción, prevención, recuperación, rehabilitación y cuidados al final de la vida (14).

Los sistemas sanitarios basados en atención primaria cumplen con cuatro atributos en la provisión de servicios: 1) Primer contacto y acceso, 2) Integralidad, 3) Longitudinalidad y 4) Coordinación. El cumplimiento de estos atributos es clave para dar respuesta pertinente a las necesidades de la población, incluyendo el manejo de enfermedades crónicas (tabla 2) (15)

\section{Modelos de cuidado de enfermedades crónicas}

Edward Wagner en 1990, propuso una sistematización para la reorganización de los servicios de salud que recibió el nombre de Modelo de cuidados crónicos (Chronic Care Model), este incluye la elaboración de protocolos y líneas de cuidado, estratificación de riesgos, educación permanente de 
equipo para el manejo de estas condiciones, división de tareas, monitoreo y definición de cuidados individualizados tomando en cuenta las preferencias y comorbilidades de cada paciente (16). En 2002, la OMS produjo una versión ampliada del modelo: el Marco de atención innovadora para afecciones crónicas (ICCC), que da al modelo un mayor énfasis a los aspectos comunitarios y políticos para mejorar la atención de salud para enfermedades crónicas (17).

El modelo de pirámide de Kayser, orientado a la gestión de casos en el cual se identifican casos de mayor complejidad (multimorbilidad o alta vulnerabilidad social), que deben ser monitoreados por un equipo o un profesional designado; identifica tres niveles de intervención según el nivel de complejidad del paciente crónico (18).

El modelo de tareas orientadas a procesos de cuidado (TOPIC) fue propuesto para afrontar los diferentes tipos de problemas de los pacientes que acuden a la consulta en primer nivel de atención; fue desarrollado por Rogers J. et al. (19), quienes categorizaron consultas prototípicas que engloban aspectos comunes en los diferentes motivos de consulta: problema nuevo, chequeo, enfermedad crónica, problema psicosocial y cambio de conducta (20). Es decir, se desarrollan los mismos procesos en la consulta, independientemente del motivo, pero variando las tareas contenidas.

Los profesionales del primer nivel de atención están en contacto próximo con los pacientes y conocen el contexto proximal y distal que influyen en la salud de sus pacientes (15). Con frecuencias las personas con enfermedades crónicas no alcanzan un óptimo cuidado a pesar de estar en contacto con servicios de salud (16). En este sentido, es de gran utilidad el manejo de herramientas que permitan sistematizar la consulta desarrollando aspectos claves de una buena comunicación y empleando óptimamente el tiempo requerido para la atención.
La reorganización de servicios, la gestión de casos y la búsqueda de un mejor abordaje individual, son claves para lograr el cuidado integral de las personas, incluyendo a las personas con enfermedades crónicas (15).

En esta revisión nos ocuparemos de describir el modelo TOPIC, usado por los médicos de familia en su práctica asistencial de abordaje individual, que provee herramientas para mejorar el cuidado de personas con problemas crónicos.

\section{Modelo TOPIC para el abordaje de problemas crónicos}

Las condiciones de salud crónicas son aquellas que tienen consecuencias recurrentes o persistentes con duración mínima de 3 a 12 meses. Su proceso causal es usualmente multifactorial y su tratamiento en la mayoría de situaciones no es curativo, sino orientado a controlar la evolución de la enfermedad y sus complicaciones y aliviar síntomas (21).

El abordaje de estos pacientes en consulta ambulatoria, no debería consistir sólo en generar la prescripción farmacológica de manera periódica o automatizada. Por ello, el modelo TOPIC para problemas crónicos, brinda una forma estructurada de atención ambulatoria, que incorpora elementos orientados esencialmente a reducir riesgos potenciales y optimizar un control sostenible en el tiempo de la enfermedad (22).

El modelo considera el uso de un lenguaje centrado en la persona, con escucha activa, considerando sus preferencias y creencias para establecer metas de tratamiento y planes de manejo en conjunto, buscando diferentes barreras potenciales para la atención (23); además consiente la utilización de estrategias y técnicas para promover el autocuidado de los pacientes, proveer educación sobre factores y estilos de vida que afecten el control de su enfermedad,

Tabla 1. Ejes prioritarios del plan de enfrentamiento contra enfermedades crónicas (13).

\begin{tabular}{ll}
\hline Eje Prioritario & Acciones \\
\hline $\begin{array}{l}\text { 1. Promoción de salud } \\
\begin{array}{l}\text { 2. Acciones de vigilancia } \\
\text { evaluación y monitoreo }\end{array}\end{array}$ & $\begin{array}{l}\text { Disminución de tabaquismo, aumento de espacios para actividades físicas, } \\
\text { promoción de alimentación saludable, disminución de consumo de alcohol, } \\
\text { etc. }\end{array}$ \\
$\begin{array}{l}\text { 3. Cuidado integral } \\
\text { Que permitan el planteamiento de metas a mediano y largo plazo. }\end{array}$ \\
$\begin{array}{l}\text { Con implementación de líneas de cuidado de los pacientes con enfermedades } \\
\text { crónicas en todos los niveles de atención con énfasis en el primer nivel) }\end{array}$
\end{tabular}




\section{REVISION DE TEMA/REVIEW}

Ramal-Moreno J. y col.

Tabla 2. Atributo de la atención primaria (15).

\begin{tabular}{|c|c|c|}
\hline Atributos & Descripción & Ventajas \\
\hline $\begin{array}{l}\text { Accesibilidad o } \\
\text { primer contacto }\end{array}$ & $\begin{array}{l}\text { Puerta de entrada al sistema } \\
\text { de salud: punto de inicio } \\
\text { de la atención y filtro para } \\
\text { acceder a los servicios } \\
\text { especializados. }\end{array}$ & $\begin{array}{l}\text { Disminuye la mortalidad y morbilidad. } \\
\text { Identificar y resolver mejor los problemas de los pacientes. } \\
\text { Mejor continuidad asistencial con el médico. } \\
\text { Al realizar un buen "filtro", menor uso de especialistas y de } \\
\text { urgencias } \\
\text { Menor porcentaje en costos globales en salud. } \\
\text { Menor uso de pruebas diagnósticas, tratamientos innecesarios. }\end{array}$ \\
\hline $\begin{array}{l}\text { Cupo y } \\
\text { longitudinalidad }\end{array}$ & $\begin{array}{l}\text { Asistencia centrada en } \\
\text { la persona a lo largo del } \\
\text { tiempo }\end{array}$ & $\begin{array}{l}\text { Facilita el cumplimiento del tratamiento de enfermedades } \\
\text { crónicas. } \\
\text { Mayor identificación de problemas asistenciales. } \\
\text { Se establecen diagnósticos más precisos y tratamientos } \\
\text { adecuados. } \\
\text { Aumenta la satisfacción de los pacientes. } \\
\text { Aumenta las posibilidades de recibir atenciones de salud } \\
\text { preventivas. } \\
\text { Menor número de hospitalizaciones y estancias más cortas. } \\
\text { Como aumentan las consultas preventivas, menos } \\
\text { enfermedades prevenibles. } \\
\text { Reducción de aproximadamente } 25 \% \text { en los costos en atención } \\
\text { en salud }\end{array}$ \\
\hline Integralidad & $\begin{array}{l}\text { Cubre todas las necesidades } \\
\text { de salud (orgánica, psíquica } \\
\text { y social). Ofrece servicios } \\
\text { preventivos y curativos, } \\
\text { refiriéndolos cuando sea } \\
\text { necesario. }\end{array}$ & $\begin{array}{l}\text { Asegura que los servicios se ajusten a las necesidades de salud } \\
\text { de la población. } \\
\text { Determina los métodos de prevención centrados en la } \\
\text { población y los separa de aquellos que son centrados a grupos } \\
\text { poblacionales específicos. }\end{array}$ \\
\hline Coordinación & $\begin{array}{l}\text { Intra Nivel/ Entre niveles } \\
\text { de atención. }\end{array}$ & $\begin{array}{l}\text { Los pacientes que reciben una atención compartida presentan } \\
\text { un número más bajo de derivaciones y desarrollan menos } \\
\text { síntomas al año de seguimiento a comparación de los demás. } \\
\text { Facilita la adecuada identificación de los problemas del } \\
\text { paciente y el buen seguimiento de éstos. } \\
\text { Incentiva la creación de un sistema informático único en salud, } \\
\text { lo cual permite una actualización constante de la información } \\
\text { del paciente; que demuestra un mejor tratamiento de la persona. }\end{array}$ \\
\hline
\end{tabular}

planes de manejo y habilidades para la resolución de problemas, que puedan mejorar sustancialmente los resultados sanitarios $(19,22-23)$.

Por otro lado, el esquema planteado por el modelo provee una guía práctica para convertir este tipo de atenciones ambulatorias en consultas organizadas, eficientes y muy útiles para el seguimiento de pacientes crónicos; favoreciendo y facilitando así la labor del médico y optimizando la intensidad de uso del sistema de salud $(19,20)$.
Para ello, el abordaje de problemas crónicos del modelo TOPIC plantea el desarrollo de cuatro tareas mayores (procesos) descritas en la tabla 3, las cuales a su vez comprenden tareas específicas para este motivo de consulta $(19,20)$, que se describen con detalle a continuación:

\section{Procesamiento de la información}

Esta tarea permite recabar una información pertinente, útil para una toma de decisiones adecuada 
en el tiempo que se dispone en el primer nivel, incluye:

a. Evaluación de las preocupaciones y expectativas del paciente: haciendo preguntas abiertas explorando sus ideas y lo que espera de la consulta (23).

b. Evaluar la gravedad y el control de la condición: preguntando por síntomas, frecuencia de uso de medicamentos de rescate, revisando los resultados de auto monitoreo en casa, observando signos en el examen físico y resultados de laboratorio que pueden reflejar gravedad o mal control $(19,22)$.

c. Evaluar la adherencia y efectos adversos al tratamiento: haciendo preguntas sobre el cumplimiento de cambios de estilos de vida, hábitos saludables; pidiendo al paciente que recuerde nombres y dosis de la medicación que usa, evaluando el recojo de medicamentos y haciendo preguntas sobre efectos adversos comunes de la medicación que toma $(19,22)$.

d. Descartar daño de órgano blanco: haciendo preguntas sobre síntomas (parestesias que podría indicar neuropatía en paciente diabético) $\mathrm{y}$ examinando signos (edema en un paciente con insuficiencia cardiaca), evaluando exámenes de laboratorio (microalbuminuria en pacientes con enfermedad renal crónica) o refiriendo a especialistas (evaluación en oftalmología para retinopatía diabética) $(19,22)$.

e. Evaluar comorbilidades: haciendo una anamnesis, examen físico y análisis de laboratorio para valorar enfermedades co-mórbidas (en un paciente diabético valorar enfermedad cardiovascular, tabaquismo, hiperlipidemia, etc.) (19-22).

\section{Desarrollo de la relación terapéutica médico- paciente}

Implica conocer a la persona y su contexto, desarrollando una relación indispensable y continua en el tiempo, con responsabilidad, que permita tener al final mejores resultados de salud.

Comprende el desarrollo de las siguientes estrategias: a) cuidado incondicional y preocupación por el paciente y su familia, b) colaboración e inclusión del paciente y su familia en el manejo de la enfermedad, c) sensibilidad a las necesidades educacionales y personales del paciente y su familia, d) una perspectiva del manejo de enfermedad crónica como un proceso en curso que requiere una relación de continuidad del cuidado entre el médico y el paciente y su familia $(19,23)$.

\section{Integración de la información y la relación médico- paciente}

Consta de 2 tareas menores:

a) Renegociar el plan de manejo: Se usa la noción que tiene el paciente sobre la enfermedad y los beneficios del tratamiento para así acordar objetivos y metas alcanzables y medibles. Se requiere de habilidades de negociación respecto de cambios en el estilo de vida. De esta manera los pacientes usualmente aceptan prestos a pruebas diagnósticas y procedimientos que le van a ayudar con tareas clave como valorar el control de la enfermedad, valorar comorbilidades, chequear daño de órgano blanco, o empezar nuevas medicaciones o hacer ajustes al régimen farmacológico lo que demanda una discusión sobre los beneficios, efectos secundarios, el

Tabla 3. Tareas del TOPIC para Enfermedad Crónica (20).

Tareas mayores

\begin{tabular}{ll}
\multicolumn{1}{c}{ Tareas mayores } & \multicolumn{1}{c}{ Tareas menores } \\
\hline \multirow{2}{*}{$\begin{array}{ll}\text { Procesamiento de la información } \\
\text { Evaluación de las expectativas del paciente. } \\
\text { Evaluar la gravedad y el control de la condición. } \\
\text { Evaluar la adherencia y efectos adversos al tratamiento. } \\
\text { Descartar daño de órgano blanco. } \\
\text { Revisar el estado de las enfermedades comórbidas. }\end{array}$} \\
$\begin{array}{ll}\text { Integración de la información y la relación } \\
\text { médico paciente }\end{array}$ & $\begin{array}{l}\text { Renegociar el plan de manejo. } \\
\text { Apoyar el autocuidado del paciente. }\end{array}$ \\
Aprendizaje de por vida & Aprender del encuentro revisando las guías clínicas aplicables
\end{tabular}


impacto psicológico de añadir otro fármaco y recordar horarios $(19,23)$.

b) Apoyar el autocuidado del paciente, los pacientes deben ser dedicados y persistentes en el cuidado de su propia enfermedad crónica. El autocuidado del paciente en la enfermedad crónica refleja las tareas de los médicos, tales como chequear el azúcar en la sangre en el hogar para evaluar la gravedad y el control, examinar los pies para daño de órgano diana, y la medición de peso para controlar una condición comórbida $(19,23)$.

\section{Aprendizaje de por vida}

Que consta de una tarea menor:

Aprender del encuentro revisando las guías clínicas aplicables, estandarizando así la práctica y manteniendo indicadores de calidad aceptable (23).

\section{DISCUSIÓN}

Existe un aumento de la prevalencia de enfermedades crónicas en el mundo a saber: enfermedades cardiovasculares, cáncer, enfermedades respiratorias crónicas y diabetes $(1,2,8)$, por eso se debe resaltar la importancia de fortalecer la respuesta del sistema de salud a las enfermedades no transmisibles en general $(12,13)$.

Los pacientes crónicos "conviven" con su enfermedad, y desarrollan a lo largo del tiempo, competencia para gestionar la nueva condición crónica, capacidad que es clave potenciar para prevenir las complicaciones $(21,24)$. En tal sentido, es necesario orientar la asistencia sanitaria durante el seguimiento a estos pacientes, hacia nuevos modelos integrados, centrados en el paciente, que potencien y fomenten el autocuidado y la corresponsabilidad para gestionar la situación de cronicidad $(15,25)$.

Se ha establecido la importancia de una buena comunicación como una piedra angular para la efectividad del seguimiento en las enfermedades crónicas en general y la diabetes en particular $(23,24)$. La comunicación con el paciente, en especial en aquellos con enfermedad crónica, requiere del desarrollo de competencias en el medico y otros profesionales de la salud, y está probado que ésta es superior cuando el médico adquiere estas habilidades de manera activa y no sólo de la experiencia (26).
Es fundamental para el control de su enfermedad, promover el rol activo que tienen las personas con enfermedades crónicas como la diabetes, a través de decisiones que toman día a día, planificación, seguimiento, evaluación y resolución de problemas relacionados con su autocuidado (24). Por ello es importante el uso de una aproximación horizontal, centrada en la persona y que valore el contexto, evitando los términos "incumplimiento" y "no adherencia" que pueden generar sentimientos de culpa en los pacientes y denotan un rol pasivo para una persona que debe "seguir las órdenes de un médico"; tal enfoque puede ayudar a minimizar la resistencia de los pacientes adoptar cambios de estilo de vida y facilitar la comunicación de estrategias de ayuda por parte de los profesionales de salud $(22,23)$.

El modelo TOPIC en el cuál se desarrolla tareas específicas que permiten abordar todos los aspectos recomendados para una buena comunicación, promueve mejores resultados en el control del paciente y nos permite un mejor manejo de los problemas en la consulta con pacientes con enfermedades crónicas (20).

El abordaje de las consultas médicas para el control de enfermedades crónicas será cada vez más común en la práctica de los sistemas sanitarios. El uso de modelos de cuidado integral centrados en las personas como el modelo TOPIC debe considerarse para la práctica de todos los profesionales de salud.

\section{Declaración de financiamiento y conflicto de intereses:}

La elaboración del artículo fue financiada por los autores. Los autores declaramos no tener conflicto de intereses.

\section{Contribución de autoría:}

JSRM y MRG: Redacción parcial de resumen, introducción, conclusiones; orden y formato del texto; búsqueda de fuentes bibliográficas. DUF y RSS: Redacción de caso viñeta, elaboración parcial de conclusiones, introducción; búsqueda de fuentes bibliográficas. MSCF: Revisión crítica importante del contenido, búsqueda de fuentes bibliográficas.

\section{Correspondencia:}

Jojay Steven Ramal Moreno

Av. Petit Thouars 1255. Departamento 1002, Cercado 
de Lima.

Correo electrónico: jojay.ramal.m@upch.pe

Teléfono: +51976764599

\section{REFERENCIAS BIBLIOGRÁFICAS}

1. Hunter DJ, Reddy KS. Noncommunicable diseases. N Engl J Med. 2013; 369:1336-1343. (Citado el 21 de agosto del 2019) Disponible en: https://www.nejm. org/doi/full/10.1056/NEJMra1109345

2. Murray CJ, Lopez AD. Measuring the global burden of disease. N Engl J Med. 2013; 369:448-457. (Citado el 21 de agosto del 2019) Disponible en: https://www. nejm.org/doi/full/10.1056/NEJMra1201534

3. Sinha DN, Mirrakhimov EM, Robinson SR, et al. Global, regional, and national age-sex-specific mortality for 282 causes of death in 195 countries and territories, 1980-2017: a systematic analysis for the Global Burden of Disease Study 2017. Lancet. 2018; 392(10159): 1736-1788. DOI: 10.1016/S01406736(18)32203-7.

4. Mathers CD, Loncar D. Projections of global mortality and burden of disease from 2002 to 2030. PLoS Med. 2006; 3(11): e442. DOI: https://doi. org/10.1371/journal.pmed.0030442

5. Omran AR. The epidemiologic transition: A theory of the epidemiology of population change. Milbank Mem Fund Q. 1971; 49:509-38.

6. Instituto Nacional de Estadística e Informática. Boletín Especial $\mathrm{N}^{\circ}$ 17: Perú: Estimaciones y Proyecciones de la Población Total, por Años Calendario y Edades Simples, 1950-2050. Lima: Instituto Nacional de Estadística e Informática; 2019. (Citado el 21 de agosto del 2019) Disponible en: http:// proyectos.inei.gob.pe/web/biblioineipub/bancopub/ Est/Lib0843/index.htm

7. The European Commission's science and knowledge service. Cost of Non-Communicable Diseases in the EU. Geneva: The European Commission's science and knowledge service ; 2017. (Citado el 21 de agosto de 2019) Disponible en: https://ec.europa.eu/ $\mathrm{jrc/en/health-knowledge-gateway/societal-impacts/}$ costs

8. Kankeu HT, Saksena P, Xu K, Evans DB. The financial burden from non-communicable diseases in low-and middle-income countries: a literature review. Health Res Policy Syst. 2013; 11:12. (Citado el 21 de agosto del 2019) Disponible en: https://health-policy-systems.biomedcentral.com/ articles/10.1186/1478-4505-11-31\#authorinformation

9. Roth GA, Huffman MD, Moran AE, et al. Global and regional patterns in cardiovascular mortality from 1990 to 2013. Circulation. 2015; 132:1667-
1678. (Citado el 21 de agosto del 2019) Disponible en: https://www.ahajournals.org/doi/10.1161/ CIRCULATIONAHA.114.008720

10. Foro de las Sociedades Respiratorias Internacionales. El impacto global de la Enfermedad Respiratoria. Ciudad de México: Asociación Latinoamericana de Tórax; 2017.

11. International Diabetes Federation. IDF Diabetes Atlas. Bruselas: International Diabetes Federation; 2017.

12. Organización Mundial de la Salud. Es hora de actuar: Informe de la Comisión independiente de alto nivel de la OMS sobre enfermedades no transmisibles. Ginebra: Organización Mundial de la Salud; 2018. (Citado el 21 de agosto del 2019) Disponible en: https://apps.who.int/iris/handle/10665/272712

13. Moehlecke-Iser BP. Estratégias preventivas para as doencas crónicas nao transmisíveis. En: Duncan B. Medicina Ambulatoria: Condutas de Atencao Primaria Baseadas em Evidéncias. Porto Alegre: Artmed; 2013. pp. 530-36.

14. Rosas A, Zárate V, Cuba M. Atributos de la Atención Primaria de Salud (A.P.S): Una visión desde la Medicina Familiar. Acta med peruana. 2013; 30 (1):42-47. (Citado el 21 de agosto del 2019) Disponible en: http://www.scielo.org.pe/scielo.php?script=sci_ar ttext\&pid=S1728-59172013000100008

15. Starfield B. Atención Primaria: equilibrio entre necesidades de salud, servicios y tecnologías. Barcelona: Masson; 2001

16. Bodenheimer T, Wagner EH, Grumbach K. Improving primary care for patients with chronic illness. JAMA. 2002; 288(14):1775-1779. doi:10.1001/jama.288.14.1775

17. Organización Mundial de la Salud. Cuidados innovadores para las condiciones crónicas: Organización y prestación de atención de alta calidad a las enfermedades crónicas no transmisibles en las Américas. Ginebra: Organización Mundial de la Salud; 2013.

18. Porter M, Kellogg M. Kaiser Permanente: an integrated health care experience. Rev Innovación Sanit y Atención Integr. 2007; 1:1-8.

19. Rogers JC, Corboy JE, Huang WY, Monteiro FM. Task-oriented processes in care (TOPIC) model in ambulatory care. New York: Springer Publishing Company Inc; 2004.

20. Masgo-Coronado KM, Cuba-Fuentes MS, MirandaMesías R. Modelo TOPIC: tareas orientadas a los procesos ambulatorios en el primer nivel de atención. Acta Med Peru. 2016; 33(3):223-7.

21. Schmidt-Duncan M. Cuidados Longitudinais e Integrais a Pessoas com Condicoes Cronicas. En: Duncan B. Medicina Ambulatória: Condutas de Atencao Primaria Baseadas em Evidéncias. Porto Alegre: Artmed; 2013. pp. 892-904. 
22. Suárez-Bustamante MA. Construyendo competencias clínicas para la atención integral de pacientes con enfermedades crónicas y estilos de vida no saludables: el enfoque de los procesos-cuidados orientados a las tareas (TOPIC). MPA e-J Med Fam Aten Prim Int. 2011; 2(5):95-106.

23. Suárez-Bustamante MA. El modelo de los procesoscuidados orientados a la Tareas en la Atención Ambulatoria (TOPIC): Tareas generales y consulta de chequeo. MPA e-J Med Fam Aten Prim Int. 2012; 1(6):3-14.

24. Diabetes Care. Standards of Medical Care in Diabetes. Diabetes Care. 2019; 42(Supplement 1):S1S2. DOI: https://doi.org/10.2337/dc19-Sint01
25. Jonkman NH. Identifying components of selfmanagement interventions that improve healthrelated quality of life in chronically ill patients: Systematic review and meta-regression analysis. Patient Educ Couns. 2016; 99(7):1087-98.

26. Fallowfield L, Jenkins V, Farewell V, et al. Efficacy of a Cancer Research UK communication skills training model for oncologists: a randomised controlled trial. Lancet. 2002; 359(9307):650-6. (Citado el 21 de agosto del 2019) Disponible en: https://www.thelancet.com/journals/lancet/article/ P I I S 0140 - 6736(02)07810-8/fulltext\# articleInformation

Recibido: 20/11/2019

Aceptado: 25/06/2020 